\title{
Antioxidant and anti-inflammatory potency of different wheat varieties and fractions
}

\author{
N. Mateo Anson ${ }^{1,2}$, R. v. d. Berg ${ }^{1}$, R. Havenaar ${ }^{1}$, G. Haenen ${ }^{2}$ and A. Bast ${ }^{2}$ \\ ${ }^{1}$ TNO Quality of Life, PO Box 360, 3700 AJ Zeist, The Netherlands and ${ }^{2}$ University of Maastricht, PO Box 616, \\ 6200 MD Maastricht, The Netherlands
}

Risk factors for diet-related disorders are oxidative stress and chronic inflammation. Wheat is a source of phytochemicals with antioxidant activity that might play a role in the observed protection of whole-grain diets against metabolic disorders.

The aims of the present study were first to investigate the bioaccessibility of antioxidant and anti-inflammatory activity from different wheat fractions during gastrointestinal (GI) transit and second to demonstrate the contribution of wheat compounds to these health factors.

Experiments were performed in the TIM system, which is a dynamic computer-controlled model consisting of gastric, duodenal, jejunal and ileal compartments simulating conditions in the human GI tract ${ }^{(1,2)}$. Samples were collected in $1 \mathrm{~h}$ aliquots for $6 \mathrm{~h}$ from the dialysates of the jejunal and ileal compartments to measure the kinetics of bioaccessibility of antioxidant capacity and anti-inflammatory responses. Antioxidant capacity (Trolox equivalent antioxidant capacity assay) and ferulic acid, polyphenol and protein contents were determined. Anti-inflammatory effects were measured in extracts and TIM samples using a human macrophage cell system with lipopolysaccharide (LPS)-induced TNF $\alpha$ and IL-6 secretion.

Antioxidant capacity was unevenly distributed within the wheat fractions (aleurone fractions $>$ bran fractions $>$ flour fractions), without differences between cultivars (Tiger and Crousty). Antioxidant capacity was correlated with the ferulic acid content $(R 0.96, P<0.00001)$. Ferulic acid was the major contributor to the antioxidant capacity in bran and aleurone fractions (50-60\%). However, ferulic acid did not reduce TNF $\alpha$ and IL-6 levels in a dose-dependent manner. The TIM experiments showed that the antioxidant capacity was bioaccessible during GI digestion. In the case of the bran and aleurone fractions the bioaccessibility was significantly lower (20-30\% initial antioxidant capacity) than that from the flour fraction. The bioaccessibility of ferulic acid was similarly low from the bran and aleurone fractions. In experiments with flour ferulic acid was below the detection limit in the dialysate samples, because of the low initial level at oral intake. The dialysates samples collected from TIM showed reduced levels of TNF $\alpha$ for the bran and aleurone fractions, reaching maximal inhibition at the 2 nd hour and 3rd hour (Figure). These data corresponded with the maximum levels of the bioaccessibility of the antioxidant capacity from these fractions.

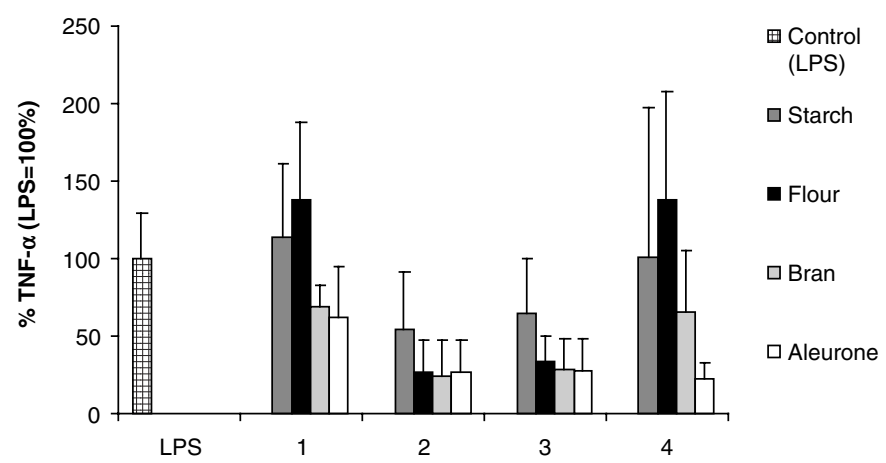

Figure. Variations in TNF $\alpha$ induced by dialysate samples from the jejunal compartment from 1st hour to 4th hour (1-4). The dialysate samples represent the absorption from the in vitro digestion of starch and the wheat fractions of the variety Tiger (flour, bran and aleurone). Values are means and standard deviations represented by vertical bars for three different days.

This publication is financially supported by the European Commission in the Communities 6th Framework Programme, Project HEALTHGRAIN (FOOD-CT-2005-514008). It reflects the authors' views and the EC is not liable for any use that may be made of the information contained in this publication.

1. Minekus M \& Havenaar R (1998) Reactor system. European Patent no. 0642382. Eur Patent Bull 98/07, Art. 97(4) and (5) EPC, dated 11.02.98.

2. Minekus M, Marteau P, Havenaar R \& Huis in 't Veld JHJ (1995) Altern Lab Anim 23, 197-209. 